\title{
Optimal Power Management for a Hydraulic Hybrid Delivery Truck
}

\author{
BIN WU, CHAN-CHIAO LIN, ZORAN FILIPI ${ }^{1}$, HUEI PENG \\ AND DENNIS ASSANIS
}

\author{
SUMMARY
}

\begin{abstract}
Hydraulic hybrid propulsion and energy storage components demonstrate characteristics that are very different from their electric counterparts, thus requiring unique control strategies. This paper presents a methodology for developing a power management strategy tailored specifically to a parallel Hydraulic Hybrid Vehicle (HHV) configured for a medium-size delivery truck. The Hydraulic Hybrid Vehicle is modelled in the MATLAB/SIMULINK environment to facilitate system integration and control studies. A Dynamic Programming (DP) algorithm is used to obtain optimal control actions for gear shifting and power splitting bet ween the engine and the hydraulic motor over a representative urban driving schedule. Features of optimal trajectories are then studied to derive i mplementable rules. System behaviour demonstrates that the new control strategy takes advantage of high power density and efficiency characteristics of hydraulic components, and minimizes disadvantages of low energy density, to achieve enhanced overall efficiency. Simulation results indicate that the potential for fuel economy improvement of medium trucks with hydraulic hybrid propulsion can be as high as $48 \%$.
\end{abstract}

\section{INTRODUCTION}

Global market competition, oil production forecasts, and environmental protection forces are stimulating work on significantly improved fuel economy of all classes of vehicles. In recent years, fuel consumed by trucks grows at a much faster rate than that of passenger cars. This is a consequence of increased proportion of light trucks and sport-utility vehicles, as well as a higher demand for ground transportation of goods. In case of trucks, the availability of new technologies for improved fuel economy is somewhat limited compared to passenger cars, due to the fact that heavier trucks already use very efficient diesel engines, as well as constraints on the potential for weight and air drag reduction imposed by payload carrying requirements. Hence, advanced hybrid propulsion systems are critical to achieving future fuel economy goals for trucks.

${ }^{1}$ Address correspondence to. University of Michigan, Department of Mechanical Engineering, Rm. 2031 W. E. Lay Automotive Lab, 1231 Beal Av., Ann Arbor, MI 48109-2133, USA. Tel.: (734) 936-0427; Fax: (734) 764 -4256; E-mail: filipi@umich.edu 
Large mass associated with trucks enables regenerating and reusing significant amounts of braking energy in hybrid powertrain configurations. Consequently, power flows through the hybrid sub system can be very high. This makes hydraulic propulsion and storage components very attractive for truck applications, since they are characterized by much higher power density compared to their electric counterparts [1]. As the energy storage device, hydraulic accumulator has the ability to accept high rates and high frequencies of charging/discharging, both of which are not favor able for batteries. However, relatively low energy density of the hydraulic accumulator requires carefully designed control strategy, so that the fuel economy potential can be realized to its fullest. Given the properties of the hydraulic components, parallel hybrid architecture is selected in this study as the most attractive and cost-effective option.

Hybridization raises the question of coordinating the operation of primary power source (typically an IC engine) and assistant power source (hydraulic motor) to maximize fuel economy while satisfying performance constraints. While there is wealth of literature focused on control of hybrid electric vehicles, publications devoted to hydraulic propulsion option are relatively scarce. Early studies attempted to use engineering intuition to devise control strategies. Buchwald and others [1] evaluated three different strategies on city buses considering simple vehicle acceleration-deceleration profiles. Wu et al. [2] proposed a strategy for passenger cars based on dividing the accumulator volume into two parts, one for regeneration and the other for road-decoupling. However, realistic in-vehicle operating conditions are varying in a very wide range, often experiencing rapid transients [3, 4]. Efficiencies of both the engine and the hydraulic pump/motor are strong functions of their respective operating conditions. Hence, optimization of control strategies requires careful consideration of duty cycles for a specific vehicle, such as the delivery truck, and goes beyond the ability of engineering intuition and static analysis. Furthermore, establishing a truly optimal benchmark is necessary for evaluating the potential of a given hybrid concept and assessing the successfulness of a particular system design.

Dynamic Programming [5] is a numerical methodology developed for solving sequential or multistage decision problems. The algorithm searches for optimal decisions at discrete points in a time sequence. It has been shown to be a powerful tool for optimal control in various application areas $[5,6,7]$. A design procedure for developing sub-optimal implementable control strategies based on Dynamic Programming (DP) was proposed previously for hybrid electric vehicles by Lin et al. [7]. Therefore, it is reasonable to expect that this approach can be successfully applied to a hydraulic hybrid propulsion system and subsequently used to improve our understanding of the tradeoffs associated with power splitting strategies and maximize overall system efficiency for hydraulic hybrid vehicles.

In this paper, we investigate application of Dynamic Programming methodology for developing power management strategies tailored for the parallel hydraulic hybrid powertrain. Next section describes modeling of the hydraulic hybrid truck in the MATLAB/SIMULINK environment. This is followed by the introduction of the 
baseline rule-based power management algorithm and illustration of its deficiencies during an elementary vehicle speed schedule. Then, the Dynamic Programming technique is employed to search for optimal trajectories of gear shifting, engine power command and motor power command. Results of DP are studied to extract the main features of the optimal control law and subsequently used to construct implementable power management rules. New control strategy is tested to demonstrate its effectiveness in further improving the fuel economy of the HHV. Quantification of individual contributions to system efficiency gains provides detailed insight into the main effects, thus offering guidelines for improving and assessing future designs. Finally, conclusions are offered in the last section .

\section{MODELING THEHYDRAULIC HYBRID VEHICLE (HHV)}

\subsection{Configuration of the HHV Medium Truck}

An International 4700 series, Class VI 4x2 truck is selected as the baseline for this work. The conventional truck has a mass of $7340 \mathrm{~kg}$ when fully loaded. In cities, medium-size trucks like the 4700 series are frequently used to undertake delivery tasks that include frequent stops-and-goes. The conventional truck $\$$ hybridized with hydraulic propulsion and storage components, and their added mass of $272 \mathrm{~kg}$ is accounted for in the simulation study.

The schematic of the proposed hydraulic hybrid version of the delivery truck's powertrain is given in Fig. 1. This rear-wheel-drive hydraulic hybrid truck has two power sources. The primary power source is the same diesel engine used in the

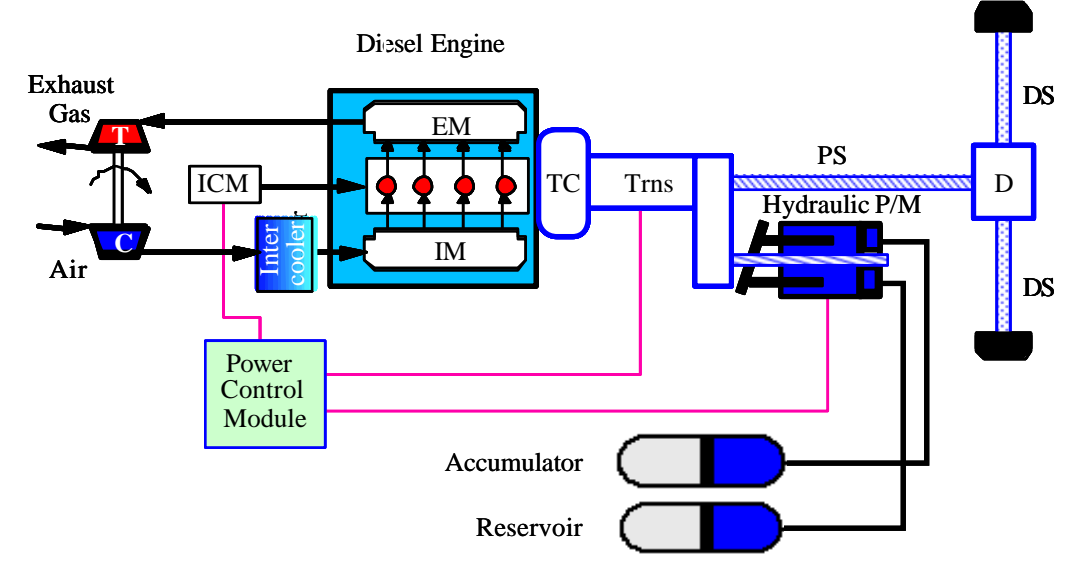

Fig. 1. The schematic of the parallel hydraulic hybrid propulsion system. 
conventional truck, that is a turbocharged, intercooled, DI Diesel V8, 7.3 L engine with rated power of $157 \mathrm{~kW} @ 2400 \mathrm{rpm}$. Although parallel hybrids offer the opportunity for engine downsizing, it is not adopted here to prevent any adverse effect on vehicle mobility and drivability when the accumulator is empty. Given hydraulic accumulator's low energy density, power assistance may not be available when the driver requires full propulsion power if the engine is downsized. Consequently, hybridization of the truck using hydraulic components aims at simultaneously improving the fuel economy and acceleration performance, while satisfying constraints related to sustained gradeability.

The Torque Converter (TC), Transmission (Trns), Propeller Shaft (PS), Differential (D) and Driving Shaft (DS) are the same as those in the conventional truck. The hydraulic pump/motor is located behind the transmission for more effective regeneration during braking The hydraulic pump/motor is coupled to a propeller shaft via a transfer case with the gear ratio of two.

The assistant power source is an axial piston pump/motor (P/M) with variable displacement. The hydraulic displacement per revolution can be adjusted via inclination of the swash plate to absorb or to produce desired torque. When pumping, hydraulic fluid flows from the low-pressure reservoir to the high-pressure accumulator; when motoring, hydraulic fluid flows in the reverse direction.

The accumulator contains the hydraulic fluid, and inert gas such as Nitrogen $\left(\mathrm{N}_{2}\right)$, separated by a piston. When hydraulic fluid flows in, the gas is compressed, and its internal energy is increased. When discharging, fluid flows out through the motor and into the reservoir. The reservoir can be regarded as an accumulator working at much lower pressure (e.g. 8.5 bar to 12.5 bar). The State of Charge (SOC) is defined here as the ratio of instantaneous fluid volume in the accumulator over the maximum fluid capacity, thus $\mathrm{SOC}=0$ corresponds to accumulator being empty, and $\mathrm{SOC}=1$ to accumulator being full.

The size of hy draulic components is configured to absorb sufficient braking energy. The hydraulic pump/motor's maximum displacement is $150 \mathrm{~cm}^{3} / \mathrm{rev}$, and its power range is $0-158 \mathrm{~kW}$, depending on the available pressure. The key specifications of the accumulator are as follows:

Fluid Capacity: 50 liters.

Maximum Gas Volume ( $\mathrm{SOC}=0)$ : 100 liters.

Minimum Gas Volume ( $\mathrm{SOC}=1)$ : 50 liters.

Pre-charge Pressure (SOC=0, at $302 \mathrm{~K}): 125$ bar.

Maximum Pressure: $=360$ bar.

\subsection{Modeling the hydraulic hybrid propulsion system}

The foundation for modeling the HHV system was the simulation of the conventional truck, previously developed at the University of Michigan Automotive Research Center. The model is implemented in the MATLAB/SIMULINK and named Vehicle-Engine SIMulation (VESIM). It has been validated against vehicle 
data measured on the proving ground [3]. Lin et al. [4] added electric components and the power management module to create Hybrid Electric VESIM . The hydraulic hybrid is modeled by replacing the electric components with hydraulic components and splitting the storage device into a high-pressure accumulator and a low -pressure reservoir, hence generating the Hybrid Hydraulic VESIM (HH-VESIM). The simulation model is forward-looking, with the source of excitation being the driver controlling the vehicle's forward speed by means of the fuel and brake pedals. The driver's command is translated by the hybrid power management module into signals defining the operation of the diesel engine, transmission and hybrid propulsion components.

The hydraulic pump/motor model is derived from the concepts proposed by Pourmovahed et al. [8]. The model accounts for both volumetric and torque losses in the pump/motor. Volumetric losses include laminar leakage loss, turbulent leakage loss and the loss due to fluid compressibility. Torque losses include effects of fluid viscosity and mechanical friction. The model captures dependency of the pump/motor efficiency on the operating mode (pumping or motoring) and operating variables, such as displacement, pressure difference and rotational speed. For example, at smaller displacements (i.e. load), both the laminar leakage and torque losses are relatively larger than at high displacements, thus reducing the pump/motor efficiency. The overall efficiency level can be varied by adjusting of model constants, thus enabling fuel economy projections for different anticipated technology levels.

For the accumulator and the reservoir, the energy conservation is applied to the nitrogen gas being compressed by the hydraulic fluid. High pressures, in excess of $35 \mathrm{MPa}$, necessitate considering real gas properties via the Ben edict-Webb-Rubin equation [9]. Due to fast compression and expansion, the gas temperature fluctuates significantly and heat transfer effects are included in the model. Since increased heat capacity generally improves accumulator efficiency, the effect of elastomeric foam added to the gas side is considered and modeled based on [10]. The internal friction and the pressure losses at the inlet/outlet and along the connecting hoses are also included in the accumulator model 8]. Typically, the accumulator average efficiency calculated based on the ratio of total energy flowing in and out is within 95 97\%.

More details about the diesel engine, driveline, vehicle dynamics and cyber driver modules are available in references [3, 4].

\section{INITIAL RULE BASED POWER MANAGEMENT STRATEGY}

As a benchmark for comparison, the control strategy previously developed for a hybrid electric (HEV) truck [4] is adopted. It is a rule-based strategy, designed with a primary goal of optimizing overall fuel economy by moving engine operating points to a more efficient region 


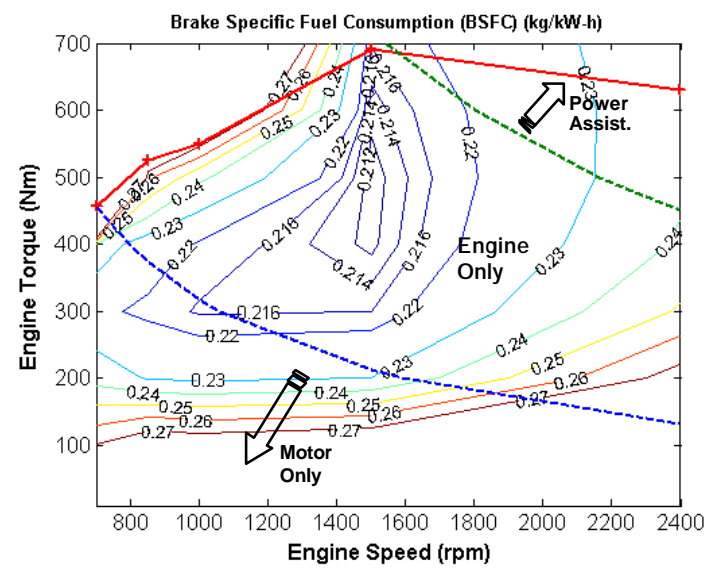

Fig. 2. Diesel engine BSFC map with constant-power lines illustrating the initial power management rules.

Fig. 2 illustrates the basic power-split idea on the engine Brake Specific Fuel Consumption (BSFC) map. The engine operating range is divided into three zones with two constant -power lines, the low - and high-power limit, respectively. When the total power demand is less than the pre-selected lower bound $(33 \mathrm{~kW})$, the hydraulic motor provides all the power, as long as there is energy available in the accumulator. Between the lower bound and upper bound $(113 \mathrm{~kW})$, the engine replaces the motor and provides the total power demand alone. Once the power requirement exceeds the upper bound, the hydraulic motor kicks-in to supply the power deficit. In case the powe command becomes larger than the sum of the upper bound $(113 \mathrm{~kW})$ and the maximum available hydraulic motor power, the engine power output is further increased to meet the total power demand. While this strategy can be effective in improving the average ef ficiency of the engine, it leads to relatively mild use of the motor. Higher power density of the hydraulic motor implies that impact of control rules will depend not only on moving the engine visitation points, but on a tradeoff between optimizing engine and motor operation.

In order to assess the effect of the initial control rules shown in Fig. 2, a simple driving schedule is designed to represent a typical acceleration-deceleration sequence (see Figure 3a). The fully loaded truck accelerates from stand-still to 35 $\mathrm{mph}$ at a rate of $0.1 \mathrm{~g}$, maintains constant speed for a short period of time, and then decelerates with the same rate until it comes to a complete stop. The motor and engine power during the transient are shown in Fig. 3b, while Fig. 3c shows accumulator state-of-charge history. Initial SOC is set at $90 \%$. Vehicle is launched with just the motor, but "low-power" limit is reached very quickly and at that point the engine replaces the motor as the sole power source. Roughly ten seconds into 
a)

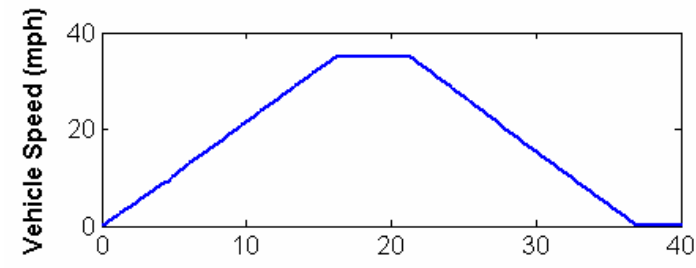

b)

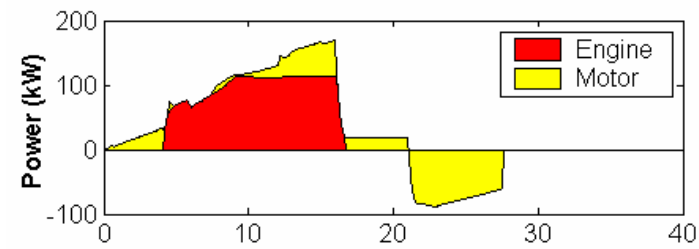

c)

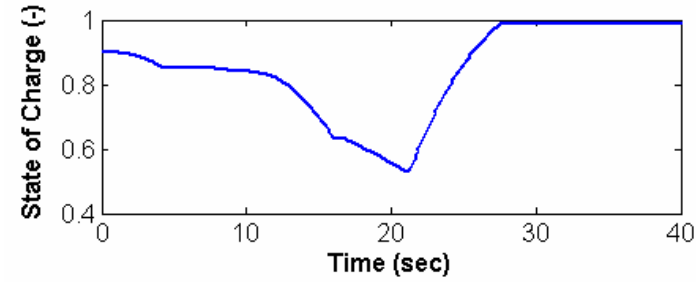

Fig. 3. Power split during an acceleration-deceleration sequence dictated by the initial power management rules.

the driving cycle, the power demand exceeds the "high-power" limit and the motor is activated again to assist the engine. Power demand for cruising is very low and is easily provided by the motor. Recalling that the maximum power of the hydraulic motor for the nominal maximum pressure in the accumulator is $158 \mathrm{~kW}$, it is obvious that initial rules under-utilize the hydraulic motor and force its operation at very low loads. Consequently, SOC in the accumulator does not drop below 50\%, and regeneration of braking energy, indicated by a negative portion of the power profile, ends early during deceleration because the accumulator becomes full. This clearly indicates inadequacies of the initial rules and motivates our work on optimizing power management specifically for the hydraulic hybrid system.

Since the braking energy is "cost-free", the rule during braking is to use regenerative braking whenever possible, i.e. whenever the hydraulic pump can supply sufficient negative torque and the accumulator is not full. Friction brakes are activated only when the braking torque requirement exceeds what the pump can provide.

In contrast to typical HEV strategies, charging directly with engine power is prohibited due to low energy density characteristic of the hydraulic accumulator. In addition, rather than attempting to sustain the SOC within narrow limits, as it is done 
with electric batteries, the accumulator state of charge can be allowed to vary from fully charged to completely empty.

Realistic assessment of vehicle fuel economy depends critically on driving conditions. The importance of the selected driving pattern is emphasized in case of hybrid vehicles. Since our focus is on a typical delivery truck, all fuel economy numbers in the remainder of this paper are evaluated over the Federal Urban Driving Schedule (FUDS).

\section{OPTIMIZATION OF POWER MANAGEMENT STRATEG Y}

\subsection{Formulation of Dynamic Programming Problem}

Once system configuration, component design and driving cycle are fixe $d$, the fuel economy depends only on strategy for splitting propulsion power between two power sources and the gear shifting logic. Since evidence present ed in Section 3 indicates deficiencies of the initial rule-based power management strategy, an optimal control problem for HHV is formulated and solved by using Dynamic Programming (DP) algorithm.

The objective is to search for optimal trajectories of control signals, $u(k)$, including engine command, hydraulic pump/motor command and gear shifting command to minimize the fuel consumption of the HHV truck over the whole driving cycle, i.e.:

$$
\min J=\min _{u} \sum_{k=0}^{k=N-1} L(x(k), u(k))
$$

where $L$ is fuel consumption over a time segment, $N$ is driving cycle length, and $x$ and $u$ are the vectors of state variables and control signals respectively. In order to match the final value of accumulator SOC with its initial value, a penalty term is added:

$$
G=\alpha(\operatorname{SOC}(N)-\operatorname{SOC}(0))^{2}
$$

Hence, the objective of the DP problem can be expressed as follows:

$$
\min J=\min _{u} \sum_{k=0}^{k=N-1} L(x(k), u(k))+G
$$

As a high -fidelity simulation tool, HH-VESIM is not suitable for DP analysis, and model simplification is conducted to reduce computation time. For Hydraulic propulsion sub-system, all dynamics are eliminated. The pressure difference between the accumulator and the reservoir is mapped as a static function of accumulator SOC, while the pump/motor efficiency model is replaced with look-up tables. More details about model simplification and DP algorithm are available from reference [7]. After simplification, only two states remain: the transmission gear number and the accumulator SOC. Based on Bellman's principle of optimality, the Dynamic Programming algorithm is presented as follows [5]: 
Step $N-1$ :

$$
J_{N-1}^{*}(x(N-1))=\min _{u(N-1)}[L(x(N-1), u(N-1))+G(x(N))]
$$

Step $k$, for $0 \leq k<N-1$

$$
J^{*}{ }_{k}(x(k))=\min _{u(k)}\left[L(x(k), u(k))+J^{*}{ }_{k+1}(x(k+1))\right]
$$

After the recursive equation is solved backwards from step $N-1$ to 0 , an optimal, time varying, state-feedback control policy can be obtained. The resulting optimal control trajectory is then used as a state-feedback controller in the simulations to generate the fuel economy result. The simulation results are shown in Fig. 4. Engine power, motor power, and gear trajectories show the optimal control actions to achieve minimum fuel consumption. The SOC trajectory starts at 0.8 and ends around 0.8 to meet the final state constraint imposed in the cost function (Eq. 3). The SOC graph in Fig. 4is characterized by large fluctuations due to high power flows through the system. However, DP optimized policy maintains SOC within limits, never allowing it to hit either upper or lower bound, and thus enabling unrestricted use of the pump/motor. The fuel economy of the DP-optimized hybrid trucks is more then doubled compared to values obtained with initial rule-based control strategy, as shown in Table 1. Large negative swings of motor power in Fig. 4 indicate effective capturing of braking energy.

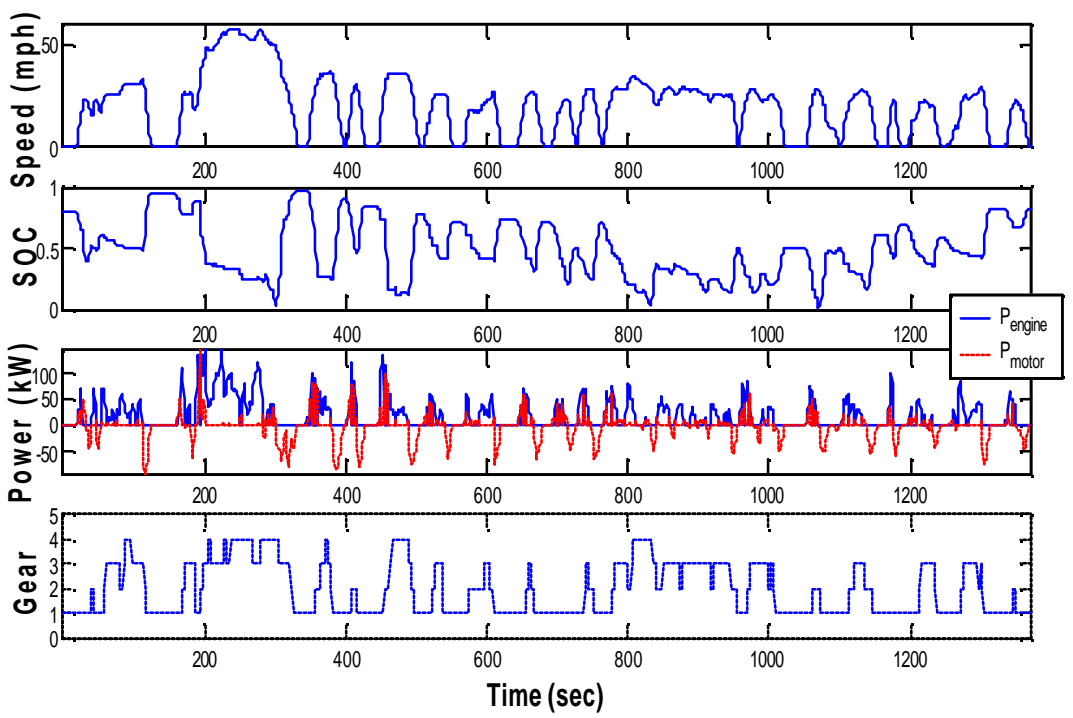

Fig. 4. Dynamic Programming results obtained over the FUDS driving schedule. 


\subsection{Improvement of Power Management Rules}

Since the Dynamic Programming algorithm is forward-looking, i.e. it uses the knowledge of the future driving conditions, the resulting optimal control signals are not applicable in practice. However, the optimal control signal trajectories provide a benchmark for evaluating applicable strategies. By analyzing the DP results, we can get useful hints about how to improve the initial power management rules and derive improved strategies that can be practically implemented.

Fig. 5 shows the gear number versus the transmission speed results produced by the Dynamic Programming algorithm. Points are nicely clustered, making it easy to locate dividing lines that separate different gear numbers. Such dividing lines represent the optimal gear shifting schedule for maximizing fuel economy. At the same time, since DP satisfies the constraint to follow the desired driving cycle, the mobility of the truck is preserved.

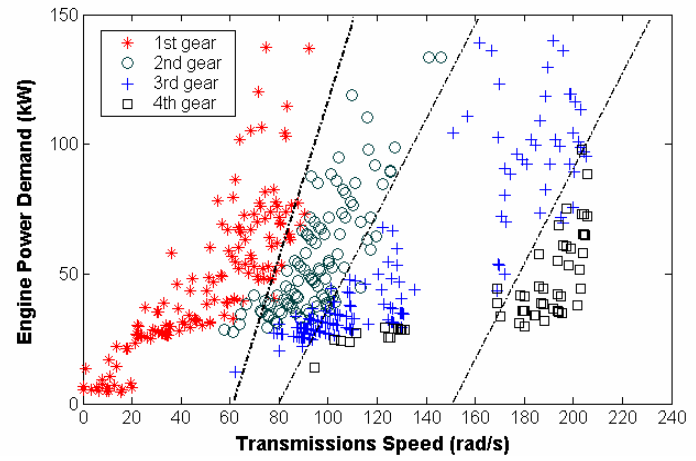

Fig. 5 Transmission gear shifting logic derived from Dynamic Programming.

Zooming-into engine and motor power histories determined by Dynamic Programming during the 400-510 second time segment of the driving schedule, (see Fig. 6), demonstrates how DP generated control policy splits the total driving power. Figure $6 \mathrm{a}$ shows the vehicle speed profiles, as well as SOC history. Figure $6 \mathrm{~b}$ illustrates engine and motor power histories. The dotted line showing instantaneous maximum motor power, determined from instantaneous motor speed and accumulator pressure, is added to help us interpret the results. Clearly, DP attempts to use the motor aggressively at the beginning of each vehicle launch. Engine power supplements a significant portion of power at launch, since demand exceeds the maximum available motor power at very low speeds associated with this event. With the exception of launch, DP tends to use the motor and engine exclusively. Whenever the total power demand exceeds maximum motor power, propulsion is switched to the engine, and in most cases the engine becomes the only source. The reason for this is found in engine and motor characteristics: bth devices have higher efficiencies at higher loads. By switching between the two power sources 
and avoiding simultaneous use of both the engine and the motor, the DP obviously attempts to keep propulsion components at high-load, high-efficiency regimes. Load of the hydraulic motor is expressed through a displacement factor, i.e. the ratio of instantaneous displacement to maximum displacement. The displacement factor time history shown in Fig. 6c demonstrates DP's effectiveness in controlling motor load. Observed behavior is in sharp contrast with the power assist rule in the initial strategy. Finally, analysis of the SOC profile in Fig. 6a indicates that DP ensures: (i) enough accumulator storage space for the future regeneration event, and (ii) appropriate hydraulic energy reserve for next launching.

a)

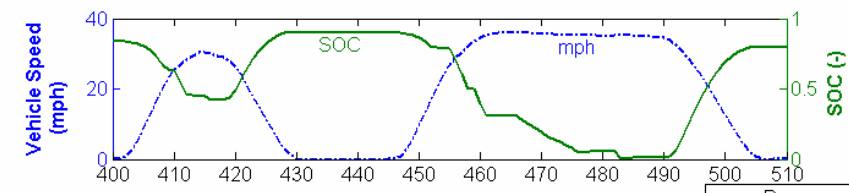

b)

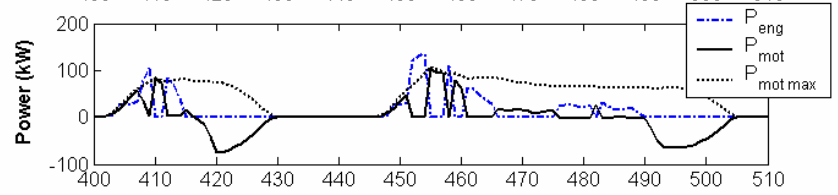

c)

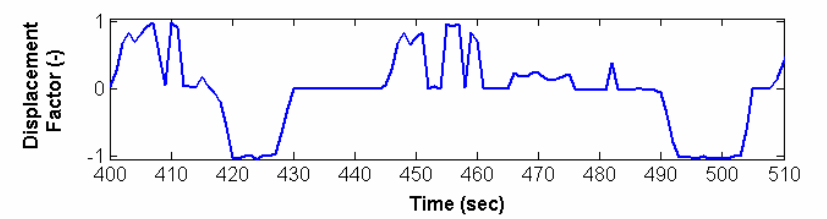

Fig. 6. DP optimized system behavior during the 400 510 second interval of the driving schedule

Unfortunately, all of these features are not practically applicable, because, unlike the Dynamic Programming algorithm, we do not know the future. In addition, very frequent switching between the motor and the engine would be unacceptable from the drivaeability standpoint. Hence, new rules have to be derived from ideas described in the previous paragraph and practical constraints. As an example, attempting to use only the motor to launch the vehicle should ensure frequent motor operation at high load and low-to-moderate speed, as well as emptying of the accumulator in preparation for the next braking event. Accounting for constrains imposed by motor power limits and availability of energy in the accumulator, leads to the following improved rules for the driving mode $\left(P_{\text {command }}>0\right)$ :

IF $S O C>0$,

$$
\begin{aligned}
& P_{\text {motor }}=\min \left(P_{\text {command }} P_{\text {motor max }}\right) \\
& P_{\text {engine }}=P_{\text {command }}-P_{\text {motor }}
\end{aligned}
$$

Else,

$$
P_{\text {engine }}=P_{\text {command }}, P_{\text {motor }}=0
$$


In summary, whenever there is energy available in the accumulator, controller will call upon the motor to satisfy the total power demand. If the power requirement is more than what motor can provide, the engine will supplement the motor power. If the accumulator is empty the engine becomes the sole power source. These rules should capture the main features of the Dynamic Programming results in a very simple and easily implementable way. Control of the braking event $\left(P_{\text {command }}<0\right)$ remains unchanged, i.e.:

$$
\begin{aligned}
& \text { IF } S O C<1 \\
& P_{\text {pump }}=-\min \left(\mid P_{\text {command }}, P_{\text {pump } \max }\right) \\
& \text { Else, } \\
& P_{\text {friction }}=P_{\text {command }}-P_{\text {pump }} \\
& P_{\text {friction }}=P_{\text {command }}, \quad P_{\text {pump }}=0
\end{aligned}
$$

\subsection{Discussion of Results}

The overall simulation results are summarized in Table 1. For each vehicle configuration, and power management option, two cases are simulated; one with High Efficiency Pump/Motor (HEPM) and the other with Low Efficiency Pump/Motor (LEPM). Thus, flexibility of the simulation is utilized to explore the boundaries for realistic systems, rather than a single arbitrary case. Hybridization significantly improves truck's fuel economy (FE) over the city driving schedule. Even with the initial generic rules, which were never optimized for HHV, the fuel economy expressed in miles per gallon (mpg) improved $32.3 \%$ (for HEPM) and $15.6 \%$ (for LEPM) compared to the conventional vehicle.

Optimized gear shifting schedule modified based on Dynamic Programming results, further improves fuel economy, but only slightly (see Table 1). However, implementation of new power splitting rules in addition to modified gear shifting, leads to FE improvements of $47.4 \%$ (HEPM) and $27.8 \%$ (LEPM). Hence, even

Table 1. Summary of simulation results obtained over the urban driving schedule

\begin{tabular}{lccccccccc}
\hline $\begin{array}{l}\text { Configur } \\
\text { ation }\end{array}$ & $\begin{array}{c}\text { Conven- } \\
\text { tional }\end{array}$ & \multicolumn{2}{c}{ Initial Rule } & \multicolumn{2}{c}{$\begin{array}{c}\text { Initial Rule }+ \\
\text { Improved Shifting }\end{array}$} & Improved Rule & \multicolumn{2}{c}{$\begin{array}{c}\text { Dynamic } \\
\text { Programming }\end{array}$} \\
\hline $\begin{array}{l}\text { P/M } \\
\text { Efficiency }\end{array}$ & NA & High & Low & High & Low & High & Low & High & Low \\
\hline mpg & 10.39 & 13.75 & 12.01 & 14.08 & 12.40 & 15.32 & 13.28 & 18.37 & 14.34 \\
\hline $\begin{array}{l}\text { mpg } \\
\text { Improve. }\end{array}$ & NA & $32.3 \%$ & $15.6 \%$ & $35.5 \%$ & $19.3 \%$ & $47.4 \%$ & $27.8 \%$ & $76.8 \%$ & $38.0 \%$ \\
\hline $\begin{array}{l}\text { Regen. } \\
\text { Energy } \\
\text { (kJ) }\end{array}$ & NA & 9748 & 9700 & 9652 & 9736 & 9459 & 9656 & 10013 & 10458 \\
\hline $\begin{array}{l}\text { Reused } \\
\text { Energy } \\
\text { (kJ) }\end{array}$ & NA & 6034 & 3187 & 5963 & 3229 & 7476 & 4524 & 8491 & 5134 \\
\hline $\begin{array}{l}\text { Reused / } \\
\text { Regen. }\end{array}$ & NA & $61.9 \%$ & $32.9 \%$ & $61.8 \%$ & $33.2 \%$ & $79.0 \%$ & $46.9 \%$ & $84.8 \%$ & $49.1 \%$ \\
\hline
\end{tabular}


though practical new rules can not achieve the same levels of fuel economy produced by the DP algorithm, they enable a very dramatic increase of HHV's ability to realize its fuel saving potential. In case the HHV is configured with the low-efficiency/low-cost motor the fuel economy improvement is almost doubled with the new power management strategy compared to initial rules.

Fig. 7 shows the differences between the initial and improved power management strategies and helps us explain the efficiency gains with new rules. With the initial strategy, the hydraulic motor provides mild power assist and operates predominantly with the small displacement factor (see Fig. 7a, bottom). With improved strategies, the hydraulic motor frequently operates with a much higher displacement factor level, often reaching full-load conditions, as shown in Fig. 7b, bottom. The combination of high load and low-to-moderate speed leads to most efficient motor operation, as shown by the efficiency iso-lines in Figure 8. For illustration purposes, motor visitation points are superimposed on the efficiency map in Fig. 8, both for initial and improved rules derived from DP results. Operating points of the motor controlled by original generic rules are clustered in the mid-speed/low-load region (displacement factor $<0.4$ ), in the area of relatively low efficiencies. The new strategy is able to move most points into the mid-speed/high -load zone characterized by highest efficiencies. In fact, new strategy enables the motor to quite frequently operate at full load (displacement factor $=1$ ). In addition, frequent use of the motor for vehicle acceleration often depletes the energy in the accumulator, which prepares the system for the next regeneration event. Therefore, situations where SOC hits the upper limit and prevents further regeneration are avoided; an example simulated with initial rules is seen in Fig. 7 a, around 425 seconds into the driving schedule.

The results in Table 1 indicate the critical role of regeneration and effective reuse of the braking energy. Total regenerated energy captured during braking reaches similar levels in all cases, except for DP calculations. However, reused energy varies significantly with both efficiency of components (HEPM vs. LEPM) and power management. The reused and regenerated energy are obtained as integrals of motoring and pumping power over the whole driving cycle. Therefore, all losses in the accumulator/reservoir, pump and motor are considered. If the hydraulic hybrid vehicle (HEPM) with initial rules is compared to the same HHV operating with optimized rules, the increase from $6034 \mathrm{~kJ}$ to $7476 \mathrm{~kJ}$ of reused energy can be attributed directly to the control strategy forcing the motor to operate in more efficient regimes. The ratio between reused energy and regenerated energy, shown in the last row of Table 1, illustrates the effectiveness of the energy conversion in the hydraulic sub-system. It should not be confused with so called wheel-to-wheel efficiencies, since the ratio does not account for losses in the driveline and friction braking. The new power management allows increase of the ratio from about $62 \%$ to $79 \%$ for the HEPM configuration, and from roughly $33 \%$ to $47 \%$ in case of LEPM configuration. In summary, regeneration of braking energy is a major factor in improving fuel economy of hybrid trucks, and hydraulic components can do it very effectively, provided that power management is optimized to take full advantage of their characteristics. 

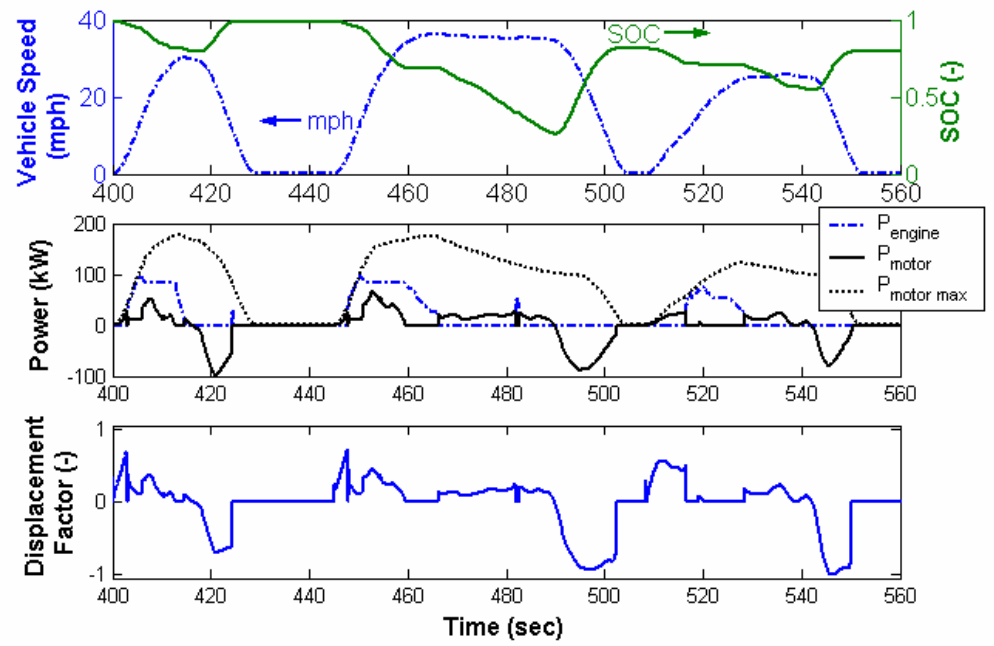

(a) Initial Rule Based
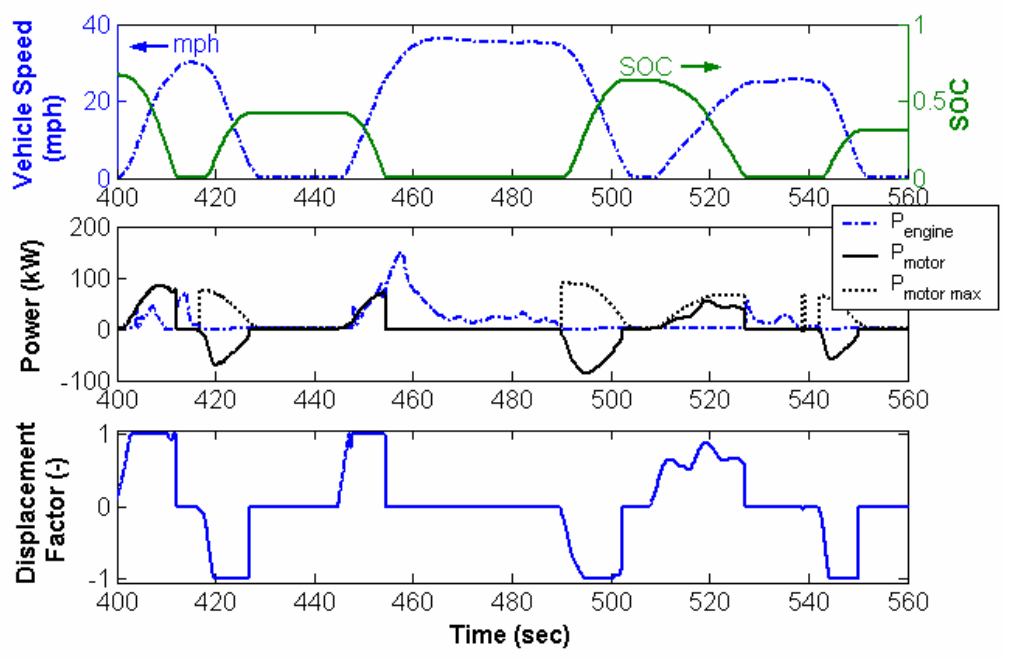

(b) Improved Rule Based

Fig. 7. Illustration of system behavior with different power management strategies during the 400 560 second interval. 


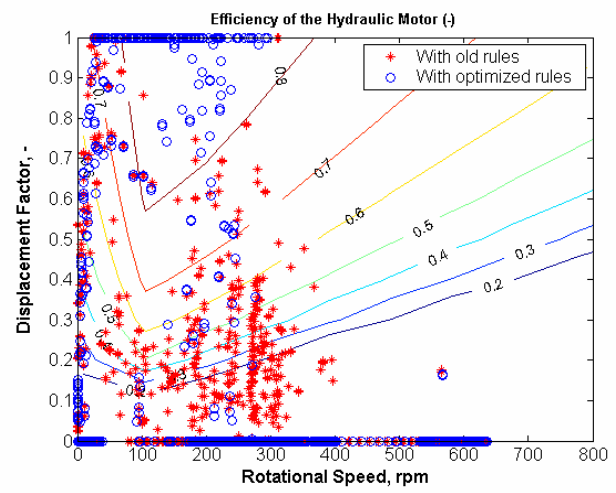

Fig. 8. Hydraulic motor operating points plotted on the motor's efficiency map (Low Efficiency PM).

\section{CONCLUSIONS}

An optimal control problem for power management of the Hydraulic Hybrid Medium Truck System is formulated and solved by using Dynamic Programming (DP) algorithm to minimize the fuel consumption. The results of forward-looking DP optimization are used to extract sub-optimal rules for power splitting and gearshifting, implementable in the practical controller. The new rules differ significantly compared to typical strategies for hybrid electric vehicles, and enable frequent use of the hydraulic motor as the sole power source during acceleration. This forces its operation at high-loads/moderate-speeds, a combination providing highest efficiency. In addition, aggressive use of the motor for vehicle acceleration often depletes the accumulator charge, thus preparing the system for the next regeneration event. Depending on the efficiency of the particular hydraulic pump/motor, the practical control strategy derived from DP results enables fuel economy increase of the HHV truck over the conventional counterpart between $\sim 28 \%$ and $\sim 48 \%$.

\section{ACKNOWLEDGMENT}

The authors would like to acknowledge the technical and financial support of the Automotive Research Center (ARC) by the National Automotive Center (NAC), and the U.S. Army Tank-Automotive Research, Development and Engineering Center (TARDEC). The ARC is a U.S. Army Center of Excellence for Automotive Research at the University of Michigan, currently in partnership with the University of Alaska-Fairbanks, Clemson University, University of Iowa, Oakland University, University of Tennessee, Wayne State University, and University of WisconsinMadison. Loucas Louca is acknowledged for his contributions to drivetrain and vehicle dynamics modeling. 


\section{REFERENCES}

1. Buchwald, P., Christensen, H., Larsen, H. and Pedersen, P.S.: Improvement of City bus Fuel Economy Using a Hydraulic Hybrid Propulsion System - a Theoretical and Experimental Study. SAE Paper 790305, Warrendale, PA, USA, 1979.

2. Wu, P., Luo, N., Fronczak, F.J. and Beachley, N.H.: Fuel Economy and Operating Characteristics of a Hydropneumatic Energy Storage Automobile SAE Paper 851678, Warrendale, PA, USA, 1985

3. Assanis, D.N., Filipi, Z.S., Gravante S., Grohnke, D., Gui X., Louca L. S., Rideout, G.D., Stein, J.L. and Wang Y: Validation and Use of SIMULINK Integrated, High Fidelity, Engine-In-Vehicle Simulation of the International Class VI Truck. SAE Paper 2000-01-0288, Warrendale, PA, USA, 2000.

4. Lin, C.C., Filipi, Z., Wang Y.S., Louca L., et al: Integrated, Feed-forward Hybrid Electric Vehicle Simulation in SIMULINK and Its Use for Power Management Studies, SAE Paper 2001-01-1334, Warrendale, PA, USA, 2001.

5. Bertsekas, D.P.: Dynamic Programming and Optimal Control, Athena Scientific, 1995.

6. Kang, J-M., Kolmanovsky, I., and Grizzle, J.W: Approximate Dynamic Programming Solutions for Lean Burn Engine Aftertreatment. Proceedings of the IEEE Conference on Decision and Control, Vol. 2, Piscataway, NJ, 1999, pp. 1703-1708.

7. Lin, C., Kang, J., Grizzle, J.W., and Peng H. : Energy Management Strategy for a Parallel Hybrid Electric Truck. Proceedings of the 2001 American Control Conference, Arlington, VA, June 2001, pp.2878-2883.

8. Pourmovahed, A., Beachley, N.H., and Fronczak, F.J: Modeling of a Hydraulic Energy Regeneration System - Part I: Analytical Treatment. Journal of Dynamic Systems, Measurement, and Control, 114 (3) (1992), pp. $155-159$.

9. Otis, D.R. and Pourmovahed, A: An Algorithm for Computing Nonflow Gas Processes in Gas Springs and Hydropneumatic Accumulators. Journal of Dynamic Systems, Measurement, and Control, 107(3) (1985), pp. 93-96

10. Pourmovahed, A., Baum, S.A., Fronczak, F.J. and Beachley, N.H.: Experimental Evaluation of Hydraulic Accumulator Efficiency with and without Elastomeric Foam. Journal of Propulsion and Power, 4(2) (1988), pp. 185-192. 